\section{A NOTE}

\section{THE TRANSMISSTON OF THE INFECTION OF TYPHOID FEVER BY OYSTERS.}

By Sir WILliaM BROADBENT, Bart., M.D., F.R.C.P., Physician-in-Ordinary to the Prince of Wales, and Senior Physician to St. Mary's Hospital.

From time to time I have seen cases of typhoid fever apparently attributable to oysters, but it is only in the course of the last autumn that the evidence of communication of typhoid by this means has been of such a character as to produce a conviction in my mind. I consider it my duty to make this evidence known, not only because it may be the means of preventing disease being thus communicated, but also because contamination of oysters by the typhoid poison can only be accidental, and is therefore probably preventable.

I saw on November 12th, 1894, with Dr. McCaskie, a young married lady who had been attended a little more than a month previously by Sir John Williams in her confinement. Up to the time of the attack, for which I was consulted, she had made a favourable recovery. The precautions against the communication of disease of any kind were more than usually careful. Milk and water were boiled, and the sanitary arrangements of the house were perfect. No other inhabitant of the house was ill in any way. In the course of her convalescence from the confinement she had oysters, and in about ten days later was attacked with typhoid fever of an unusually severe type, with early acute tympanites, which proved fatal.

On December 6th I saw, with Dr. Benson, a young gentleman who, three weeks before, had had an attack of influenza. After subsidence of the fever he was kept in his room on account of bronchial catarrh. During this time he partook freely of oysters. The sanitary arrangements of the house were faultless; no other member of the household-a large nnesuffered; no other source of the attack of typhoid fever, from which he suffered, could be traced.

On November 27 th I saw, with Mr. Manley Sims, a gentleman and his wife who had simultaneously been attacked with typhoid fever. The sanitary surroundings were irreproachable; no possible source of typhoid fever could be traced, except that a fortnight before they had partaken of oysters. The simultaneous attack was strong evidence of simultaneous exposure to the poison. One of these cases ran a somewhat severe course ; the other, in respect of fever, was mild, but was attended with more than usual gastric catarrh. In the course of our attendance on these cases $\mathbf{M r}$. Manley Sims gave me particulars of an extremely severe attack of typhoid fever in a c'ild, aged 8. No other member of the household was affected, and no possible source of poisoning could be traced, until it was ascertained that shortly before the attack the child had eaten twelve oysters at the house of friends of the parents.

On November 23rd I saw a young married lady who, ten or fourteen days previously, had on two occasions had six oysters with a young married lady of about her own age, a cousin. She had a mild attack of typhoid fever, attended, however, with unusually severe gastric catarrh. Her cousin, who meantime had gone to Italy, also suffered from typhoid fever.

On November 19th, 1894, I saw, with Dr. English, two young men living in the same house, one being the brotherin-law of the other. The sanitary arrangements of the house were good. No other member of the household suffered. The two young men were engaged together in the City in irreproachable surroundings. They had simultaneously been taken ill with an unusually severe form of typhoid fever. One was moribund at the time of our first consultation; the other, after apparently recovering from the severe symptoms, - of which acute tympanites was one ultimately died of perfora: tion. No cause for the disease could be discovered, except that after visiting a theatre they had had an oyster supper together some ten ri.iss previous to the setting in of the symptoms.

Early in December I was called in consultation into the country by Dr. Forster of Daventry to see a clergy- man and his daughter, 12 years of age, both suffering from typhoid fever. The daughter had been attacked a few days before her father. Typhoid fever was an unknown disease in the neighbourhood, the sanitary arrangements were those usually found in a large country house. Great attention had been paid to the ventilation of the drains and cess pit. No other member of the household was ill. About a fortnight before the attack they had twice had oysters down from London, which had been eaten by the father and the little girl, no one else having partaken of them.

Other cases in which the evidence was equally convincing have come to my knowledge although the patients have not been under my care, and I have seen several cases of typhoid fever in gentlemen engaged in the city, their families at home not suffering. On inquiry it has been found that they frequently took oysters for lunch.

\section{ON THE PERSISTENCE OF THE BACILLUS OF LOEFFLER AFTER RECOVERY FROM DIPHTHERIA.}

Bx E. A. SCHÄFER, F.R.S.,

Professor of Physiology in University College, London.

The following facts are of interest in connection with the persistence of the Loeffler bacillus after diphtheria, and the effects of antoxic serum in this disease.

On November 28th, 1894, in conjunction with Dr. Muspratt and Mr. McGillicuddy, of Bournemouth, I administered $10 \mathrm{c.cm}$. of antitoxin, furnished by Dr. Ruffer, to each of two school-boys (aged 13) suffering from diphtheria. The clinical appearances were unmistakable. and were confirmed by bacteriological investigation. The dose was repeated in twenty-four hours, by which time a marked improvement was manifest in both cases, and in another twenty-four hours the membrane had totally disappeared in one case, and almost so in the other. To hasten its removal this second boy received a third injection of $10 \mathrm{c.cm}$. on the morning of November 31st, this time with antitoxin of Dr. Klein's preparation. By the same evening no trace of membrane remained, and both boys were practically convalescent. Their recovery has been uninterrupted, but the one boy who received only $20 \mathrm{c.cm}$., and who was also not treated until the sixth day of the disease, had some albuminuria during convalescence, and has since had slight paralysis, whereas the other boy, who received in all $30 \mathrm{c} \mathrm{cm}$, and was treated on the third day of the disease, has had no albumen, and up to the present has shown no symptoms of paralysis.

Samples of the tonsillar mucus were sent from time to time to Dr. Ruffer for examination, and up to four weeks from the disappearance of the membrane they afforded on cultivation abundant colonies of the Loeffler bacillus. After four weeks these showed "many involution forms," and a sample taken from one of the cases at five weeks after the disappearance of the membrane no longer furnished evidence of their presence.

Another boy in the same school had an attack of "tonsillitis" early in May last, which was only recognised as diphtheritic on the appearance two months later of characteristic paralyses. A sample of mucus from this boy's throat was sent seven months and a-half after the attack, along with the others to the British Institute of Preventive Medicine for comparison with them, and was found to present numerous colonies of the typical Loeffler bacillus in serum cultivations; these cultivations have since been used successfully for inoculation of animals. It will be interesting to determine how much longer the organism continues to manif $r$ st itself in this case, and whether in cases treated by antitoxic serum the period is reduced; in the meantime the fact of a persistence of pathologically active bacilli already extending to seven months and a-half furnishes food for reflection. As regards a possible connection between the "May" and the "November" cases it may be noted that one of the "November" cases slept in the same dormitory, and the other one shared a desk with the "May" case.

The sanitary arrangements of the school are excellent. and both drinking water and milk are always boiled. There have been no other cases of diphtheria either in the schnol or 